\title{
Caracterização de Fatores de Risco para o Comportamento Criminal em Detentos
}

\author{
Germano Gabriel Lima Esteves' \\ Bruna Nogueira Romariz Barros² \\ Gustavo Henrique Silva de Souza ${ }^{3}$ \\ Jorge Artur Peçanha de Miranda Coelho \\ ${ }^{1}$ Universidade de Rio Verde (UniRV) - Rio Verde, GO \\ ${ }^{2}$ Universidade Federal de Alagoas, Maceió, AL \\ ${ }^{3}$ Instituto Federal do Norte de Minas Gerais, Teóflio Otoni, $M G$
}

\begin{abstract}
Resumo
O presente estudo teve como objetivo caracterizar fatores de risco, especificamente, a psicopatia, agressividade e a personalidade, em detentos brasileiros inseridos em atividade de ressocialização. Para tanto, contou-se com a participação de 48 detentos do sexo masculino, em regime fechado de uma unidade prisional, com idade média de 34,6 anos (DP = 8,68). Os seguintes instrumentos foram aplicados: (1) Questionário de Agressão de Buss-Perry;(2) Inventário dos Cinco Grandes Fatores da Personalidade - IGFP - 5; (3) Levenson Self-Report Psychopathy scale; (4) Questionário Demográfico. Comparando com a literatura, os resultados revelaram um perfil divergente do tipicamente reportado, indicando baixa hostilidade, psicopatia primária e uma personalidade caracterizada pela amabilidade e abertura a mudança. Observou-se ainda que o tempo de inserção em atividade de ressocialização não guarda relações com os construtos avaliados. Discute-se o padrão inesperado comparando sua divergência da literatura e aponta-se para avaliação dessa população por métodos de controle de desejabilidade social.
\end{abstract}

Palavras-chave: psicopatia, personalidade, agressividade, fatores de risco, detentos

\section{Characterization of Risk Factor Criminal Behavior in Convicts}

\begin{abstract}
The present study aims to characterize the risk factors, specially psychopathy, aggressiveness and personality, in Brazilian convicts inserted in resocialization activities. Participants included 48 male convicts with a mean age of 34.6 years $(\mathrm{SD}=8.68)$ serving time in closed regime in a prison unit. The following instruments were applied: (1) Aggression Questionnaire BussPerry; (2) Inventory of the Big Five Personality - IGFP - 5; (3) Levenson Self-Report Psychopathy Levenson Scale; and (4) Demographic Questionnaire. Compared with the literature, the results revealed a divergent profile from the typically reported, indicating low hostility, primary psychopathy and a personality characterized by amiability and openness to change. It was also observed that the time spent in resocialization activities was not related with the evaluated constructs. The unexpected pattern is discussed by comparing its divergence from the literature and we suggest the evaluation of this population by methods of social desirability control.

Keywords: Psychopathy; personality; aggression; risk factors; convicts
\end{abstract}

Caracterización de los Factores de Riesgo en el Comportamiento Criminal de Detenidos (Presos)

\section{Resumen}

Este estudio tuvo como objetivo caracterizar factores de riesgo, especificamente, psicopatía, agresividad y personalidad, en presos brasileños insertados en actividades de resocialización. Participaron 48 detenidos, con una edad media de 34,6 años (DE $=8,68$, que estaban en una cárcel cumpliendo pena en régimen cerrado. Se aplicaron los siguientes instrumentos: (1) Cuestionario de Agresión de Buss-Perry, (2) Inventario de los Cinco Grandes Factores de la Personalidad - IGFP - 5; (3) Levenson Self-Report Psychopathy Scale, y (4) el Cuestionario Demográfico. Comparados con la literatura, los resultados revelaron un perfil divergente de lo típicamente reportado, indicando baja hostilidad, psicopatía primaria y una personalidad caracterizada por la amabilidad y abierta al cambio. Se observó además que el tiempo de inserción en actividades de resocialización no guarda relación con los constructos evaluados. Se discute el modelo inesperado comparando su divergencia de la literatura y se apunta hacia la evaluación de esa población por métodos de control de deseabilidad social.

Palabras-clave: psicopatía; personalidad; agresividad; factores de riesgo; detenidos

\section{Introdução}

Com 548 mil detentos, o Brasil aparece em quarto lugar entre as maiores populações carcerárias do mundo e, em primeiro, dentre os países da América do Sul (Walnsley, 2013). Esse cenário torna-se ainda mais grave com o aumento significativo desse contingente ao longo dos últimos anos, passando de 232.755, em 2000, para 548.000, em 2013, e chegando, segundo o último relatório do Levantamento 
Nacional de Informações Penitenciárias (Infopen), a 622.202, em 2014, e atingindo uma taxa de 306,2 detentos a cada 100.000 habitantes (Walnsley, 2013; Brasil, 2014). Em decorrência dessa superpopulação, a capacidade do sistema prisional brasileiro, que é de cerca de 300 mil detentos, apresenta um déficit de vagas no sistema prisional que passa de 300 mil vagas (Brasil, 2014).

Diante disso, o Sistema Integrado de Informações Penitenciárias (InfoPen) do Ministério da Justiça, aponta os atrasos no sistema judiciário como um dos grandes responsáveis para a superlotação, registrando $40 \%$ dessa população carcerária como provisória, ou seja, detentos que não tiveram condenação em primeiro grau (Brasil, 2014). Posto isso, o Conselho Nacional de Justiça apresentou como uma de suas metas julgar a maior quantidade de processos, englobando a concessão de benefícios penais em atraso, como a progressão de regime penal, que confere a detentos o direito de cumprir suas penas em regime semiaberto ou em liberdade condicional (Brasil, 2015). É nesse contexto que se faz necessária a avaliação de fatores de risco para o comportamento criminal, ou seja, fatores que de fato elevam a probabilidade do indivíduo a reincidir criminalmente (Huss, 2011).

Nesses termos, a avaliação de fatores de risco para o comportamento criminal tem sido objeto de diversos estudos no mundo todo, construindo um vasto e consistente corpo teórico e identificando construtos, como a agressividade (Tóth, Halász, Mikics, Barsy, \& Halle, 2008; Hershcovis et al., 2007), a psicopatia (Penney \& Moretti, 2007) e os traços de personalidade desviantes: impulsividade, neuroticismo e busca de sensações (Miller, Lynam, \& Leukefeld, 2003; Sobral, Romero, Luengo, \& Marzoa, 2000). Não obstante, no Brasil, em específico, poucos são os estudos que buscaram identificar ou compor um perfil dos fatores de risco presentes na população carcerária (Schmitt, Pinto, Gomes, Quevedo, \& Stein, 2006; Jozef, Silva, Greenhalgh, Leite, \& Ferreira, 2000; Armani \& Cruz-Silva, 2010; Vargas, Hoffmeister, Prates, \& Vasconcellos, 2015). Como um possível resultante dessa carência teórica, pode-se apontar a proliferação do emprego de instrumentos de avaliação psicológica, que reúnem poucas evidências empíricas, no âmbito da prática em psicologia forense (Silva \& Fontana, 2011; Lago \& Bandeira, 2008). Nesse sentido, serão tratados, nos próximos tópicos, os construtos elencados neste estudo para avaliação de detentos e sua constituição como fator de risco.

\section{Psicopatia}

A psicopatia pode ser definida como um conjunto de traços disruptivos de personalidade envolvendo aspectos interpessoais (por exemplo, senso de grandiosidade e loquacidade), afetivos (ausência de remorso e afeto superficial) e comportamentais (comportamento impulsivo e antissocial) (Hare, 2013; Schraft, Kosson, \& Mcbride, 2013). Mais especificamente, a psicopatia pode ser caracterizada por uma gama de características, como loquacidade/charme superficial, egocentrismo e grandiosidade, ausência de remorso ou culpa, falta de empatia, mentira patológica/manipulação, insensibilidade afetivo-emocional, impulsividade, descontroles comportamentais, necessidade de estimulação/tendência ao tédio, irresponsabilidade, problemas de comportamento precoce e comportamento adulto antissocial (Hare, 2013). Nesse contexto, o instrumento mais difundido para a avaliação da psicopatia é a escala Psychopathy Checklist-Revised (PCL-R; Hare, 1991, 2003), utilizada no contexto brasileiro por diferentes estudos com detentos (Vargas et al., 2015; Jozef et al., 2000).

Entretanto, alguns estudos têm questionado a adoção do comportamento criminoso ou antissocial como parte da definição de psicopatia, argumentando que esta deve ser compreendida como traços de personalidade que são socialmente indesejáveis, colocando o comportamento criminoso como um correlato (Hauck Filho, Texeira, \& Dias, 2012). Nessa proposição, outras medidas têm sido elaboradas, como é o caso da Levenson Self-report Psychopatby Scale (SRPS), que compreende uma medida de autorrelato, baseada nos critérios da PCL-R, e composta por 26 itens divididos em dois fatores: (1) Psicopatia primária, projetada para avaliar uma postura egoísta, insensível e manipuladora para com os outros, e (2) Psicopatia secundária, elaborada para avaliar a impulsividade e o estilo de vida inapropriado (Levenson, Kiehl, \& Fitzpatrick, 1995). Em vez de enfatizar a atividade criminosa, a SRPS foi projetada para obter informações sobre comportamentos mais típicos da vida social, como a integridade do indivíduo. Assim, diversos estudos têm se utilizado da SRPS para reunir evidências em populações carcerárias (Smith, Selwyn, Wolford-Clevenger, \& Mandracchia, 2014; Brinkley, Diamond, Magaletta, \& Heigel, 2008; Levenson, 2004). No Brasil, até o ano de 2016, a única utilização desse instrumento foi com objetivo de verificar as propriedades psicométricas para o contexto brasileiro, utilizando uma amostra de universitários (Hauck Filho \& Teixeira, 2014). No presente estudo, optou-se pela utilização da 
SRPS, admitindo o comportamento criminoso como um correlato da psicopatia.

Assim como o traço de psicopatia, que tem sido amplamente apontado por diversos estudos como um fator de risco para o comportamento criminal (Penney \& Moretti, 2007; Swogger, Walsh, Lejuez, \& Kosson, 2010), alguns traços normais da personalidade também têm sido apontados como fatores de risco, como neuroticismo e abertura à mudança (Miller et al., 2003; Sobral et al., 2000).

\section{Traços de Personalidade}

Diante dos resultados alcançados em várias culturas, o modelo dos CGF tem sido amplamente utilizado em vários estudos para explicação de diversos comportamentos. Nesse modelo, McCrae e Costa (1992) identificaram cinco traços de personalidade considerando-os como tendências básicas com fundamento biológico, que não são influenciadas diretamente pelo ambiente. Embora existam algumas divergências quanto à tradução de cada uma dessas dimensões adotar-se-á aqui as seguintes nomenclaturas: abertura à mudança (openess to experience); conscienciosidade (conscientiousness); extroversão (extraversion); amabilidade (agreeableness); e neuroticismo (neuroticism).

Nesse contexto, a constituição da personalidade como fator de risco tem sido evidenciada em diversos estudos (Listwan, Piquero, \& Voorhis, 2010; Vachon et al., 2013). Entretanto, no contexto brasileiro, a caracterização da personalidade como fator de risco, em sua maioria, tem reunido evidências em amostras de universitários relacionando as dimensões do CGF à psicopatia ou aos comportamentos antissociais (Formiga, Omar, \& aguiar, 2010; Monteiro et al., 2015). No geral, o perfil de relacionamento do traço de psicopatia com as dimensões dos CGF é consistentemente apresentado em uma metanálise realizada por O`Boyle, Forsyth, Banks, Story e White (2014) que contou com 215 estudos que visaram avaliar os CGF no contexto da tríade sombria. Os resultados apontaram o traço de psicopatia associado negativamente com as dimensões de amabilidade $(r=$ $-0,42)$ e conscienciosidade $(r=-0,31)$, e positivamente relacionado com as dimensões de extroversão $(r=$ $0,04)$, neuroticismo $(r=0,05)$ e abertura a mudança $(r$ $=0,04)$. Contudo, esse padrão não é absoluto, Monteiro et al. (2015) reportam um padrão diferente ao relacionar as dimensões do CGF com o escore total do Medida Triádica da Psicopatia reportando uma correlação positiva e estatisticamente significativa com a abertura à mudança $(r=0,21)$, extroversão $(r=0,24) \mathrm{e}$

Psico-USF, Bragança Paulista, v. 23, n. 4, p. 719-730, out./der. 2018 relações sem significância estatística com neuroticismo $(r=0,02)$ e conscienciosidade $(r=0,0)$, sendo a amabilidade o único relacionamento que corroborou com a literatura $(r=-0,17)$.

Apesar da escassez de estudos sobre a caracterização das dimensões da personalidade na população carcerária brasileira, as evidências da composição da personalidade como explicadora de condutas antissociais ou criminosas são robustas, apontando também para sua relação com outros fatores de risco, como o traço de psicopatia e a agressividade (Formiga, Cavalcante, Araújo, Lima, \& Santa, 2007).

\section{Agressividade}

A agressividade apresenta-se como qualquer comportamento dirigido para um outro indivíduo que é realizado com a intenção imediata de causar dano, de modo que o autor deve acreditar que o comportamento vai prejudicar o alvo e este deve estar motivado para evitar o comportamento (Bushman \& Anderson, 2001). Nesse contexto, Buss e Perry (1992) propuseram uma manifestação multidimensional da agressão, composta por agressão física, agressão verbal, hostilidade e raiva. As agressões física e verbal compreendem o componente moto, instrumental da agressão, a raiva representa o componente fisiológico, emocional do comportamento agressivo, e a hostilidade abrange o componente cognitivo, representando o sentimento de desconfiança em relação ao próximo (Buss \& Perry, 1992).

Nessa perspectiva, Diamond e Magaletta (2006) avaliaram a agressividade em uma amostra de 603 detentos, reportando uma média (M) de 2,24 e desvio padrão (DP) de 0,42 para o escore de agressividade geral, $M=$ 2,43 e $D P=0,49$ para agressividade física, $M=2,08$ e $D P$ $=0,31$ para agressividade verbal, $M=1,85$ e $D P=0,42$ para o fator raiva e $M=2,61$ e $D P=0,47$ para o fator hostilidade. Assim, a proposição da agressividade como um fator de risco para o comportamento criminoso tem encontrado suporte em diferentes estudos (Brinkley et al.., 2008, Schippers, Märker, \& Fuentes-Merillas, 2001). Não obstante, a caracterização da agressividade no contexto carcerário brasileiro ainda encontra-se defasada e os estudos que apontam o relacionamento com comportamentos antissociais ou condutas delitivas se utilizam de amostras de universitários ou adolescentes. Nesse cenário, Formiga, Cavalcante, Araújo, Lima e Santana (2007) avaliaram a relação entre agressividade e o traço de abertura à mudança em uma amostra de 504 estudantes, relatando $M=2,75$ e $D P=0,91$ para o escore de agressividade geral, $M=2,53$ e $D P=0,80$ 
para agressividade física, $M=2,87$ e $D P=0,98$ para agressividade verbal, $M=2,61$ e $D P=0,97$ para o fator raiva e $M=2,99$ e $D P=0,90$ para o fator hostilidade.

Diante do exposto, o presente estudo tem por objetivo caracterizar fatores de risco, em específico, a psicopatia, agressividade e a personalidade, em detentos brasileiros inseridos em atividade de ressocialização, bem como avaliar a relação entre o tempo de inserção na atividade de ressocialização e os escores apresentados nos construtos elencados. Assim, espera-se contribuir teoricamente com a apresentação de evidências empíricas na caracterização de uma amostra carcerária e de modo prático, reportando indicações para avaliações no contexto forense.

\section{Método}

\section{Participantes}

Participaram 48 detentos do sexo masculino em regime fechado, de uma unidade prisional em Maceió - AL, inseridos em atividade de ressocialização e com idade média de 34,6 anos ( $D P=8,68)$. Essa amostra foi de conveniência (não probabilística) onde participaram os indivíduos, que estavam presentes e sendo solicitados a colaborar, concordaram em fazer parte da pesquisa. A Tabela 1 apresenta a caracterização dos participantes.

\section{Instrumentos}

(1) Questionário de Agressão de Buss-Perry: Elaborado por Buss e Perry (1992) e validado por Gouveia, Chavez, Peregrino, Branco e Gonçalves (2008). O questionário é composto por 26 itens que avaliam a agressão, em quatro dimensões, a saber: Agressão Física $(\alpha=0,65)$ composto por sete itens ("Se alguém me bater, eu bato nele de volta"); Agressão Verbal $(\alpha=0,52)$ composto por três itens ("Não consigo ficar calado(a) quando as pessoas discordam de mim"); Raiva $(\alpha=0,71)$ que apresenta seis itens ("Alguns amigos dizem que sou cabeça quente"); e, Hostilidade $(\alpha=0,62)$ composto de 11 itens ("Quando as pessoas são muito gentis, duvido de suas intenções”). Os itens são respondidos em uma escala de cinco pontos, tipo Likert, com os seguintes extremos: $1=$ discordo totalmente e $5=$ concordo totalmente.

(2) Inventário dos Cinco Grandes Fatores da Personalidade - IGFP - 5 (versão curta): Elaborado por John, Donahue e Kentle (1991), adaptado e validado para o contexto brasileiro por Andrade (2008). Formado por 32 itens estruturados em sentenças curtas, distribuídos entre cinco fatores: Abertura $(\alpha=$ $0,65)$, composto por nove itens, como "É original, tem sempre novas ideias"; Conscienciosidade $(\alpha=0,65)$, composto por seis itens, como "Faz as coisas com eficiência"; Extroversão $(\alpha=0,75)$, composto oito itens, como "Tende a ser quieto, calado"; Agradabilidade $(\alpha=0,69)$, composto por três itens, como "Gosta de cooperar com os outros" e; Neuroticismo $(\alpha=0,75)$, composto por seis itens, como "Fica nervoso facilmente". Os itens são respondidos em uma escala de

Tabela 1

Caracterização da Amostra - Dados Sociodemográficos

\begin{tabular}{cc}
\hline dados sociodemográficos & distribuição \\
\hline Cor & \\
branco & $11(22,9 \%)$ \\
preto & $23(47,9 \%)$ \\
pardo & $14(29,2 \%)$ \\
\hline escolaridade & \\
analfabeto & $20(41,7 \%)$ \\
fundamental incompleto & $14(29,2 \%)$ \\
fundamental completo & $3(6,3 \%)$ \\
médio incompleto & $6(12,5 \%)$ \\
médio completo & $4(8,3 \%)$ \\
superior completo & $1(2,1 \%)$ \\
tempo em atividade de ressocialização* & 0,25 a $15(M=2,42 ; D P=2,74)$ \\
\hline
\end{tabular}

Nota. *Variáveis calculadas em anos. 
cinco pontos, variando de $1=$ discordo totalmente a $5=$ concordo totalmente.

\section{(3) Levenson Self-Report Psychopathy scale}

- LSRS: Elaborado por Levenson et al. (1995) e adaptado e validado por (Hauck-Filho \& Teixeira, 2014). Composta por 26 itens distribuídos em dois fatores: Psicopatia primária $(\alpha=0,83)$ composto por 17 itens e psicopatia secundária $(\alpha=0,68)$ composto por nove itens. Os itens são respondidos em uma escala de cinco pontos, variando de $1=$ discordo totalmente a $5=$ concordo totalmente.

(4) Questionário Demográfico: Com perguntas, como idade, sexo, estado civil e escolaridade. Além disso, também foram incluídas questões, como tipificação criminal autorrelatada, tempo de inserção na atividade de ressocialização, tempo da sentença e tempo já realizado da sentença.

\section{Procedimentos}

Inicialmente, foi solicitada a autorização para a vara de execuções penais e para a diretoria do presídio em que a pesquisa foi realizada. Logo após, o projeto foi submetido ao Comitê de Ética em Pesquisa por meio da Plataforma Brasil, tendo sido aprovado (Parecer $\left.n^{\circ}: 398.042 / 2013\right)$, a aplicação desses instrumentos foi realizada por meio de entrevista cara a cara em sala com condições ótimas e seguindo o modus operandi do sistema prisional. Utilizou-se um procedimento padrão, procurando garantir o mínimo de respostas enviesadas. A aplicação foi realizada por dois pesquisadores que interferiram o mínimo possível no processo de aplicação, evitando emitir comentários ou dar respostas que pudessem induzir os participantes. Além disso, os participantes foram esclarecidos sobre o anonimato e sigilo de suas respostas e obteve-se o consentimento livre e esclarecido de todos os participantes por meio da assinatura de um Termo de Livre Consentimento. Também foi garantido o caráter voluntário da participação, bem como o respeito às diretrizes éticas que regem a pesquisa com seres humanos.

\section{Análise de Dados}

Por meio do software SPSS 23, foram realizadas estatísticas descritivas (média, desvio padrão, mínimo e máximo, erro padrão e intervalo de confiança de 95\%) para descrição dos resultados em particular de cada uma das escalas e seus respectivos fatores. Após, utilizou-se o teste Kolmogorov-Smirnov para verificar a distribuição das variáveis. Assim, para as variáveis com distribuição normal, foram realizadas análises de correlação $r$ de Pearson e, para aquelas que não apresentaram distribuição normal, utilizou-se o coeficiente de correlação rô de Spearman.

\section{Resultados}

Inicialmente, procederam-se análises descritivas de cada uma das escalas utilizadas e de seus respectivos fatores. Como mostrado na Tabela 2, os índices de agressividade explicitam um escore geral próximo do ponto médio da escala, sendo que os fatores de agressividade hostil, verbal e raiva apresentaram-se de modo mais característico, enquanto que a agressividade física apresentou o escore mais baixo. Ainda na Tabela 2, são apresentados os escores referentes aos traços de personalidade, onde pode-se observar que, tipicamente, a amabilidade apresenta-se como o traço mais predominantes, seguida pelo traço de abertura à mudança, conscienciosidade, extroversão e, como a única dimensão com escore inferior ao ponto médio da escala, o neuroticismo. No tocante à psicopatia, também na Tabela 2, o escore geral da amostra estudada apresenta uma pontuação mediana em psicopatia, do mesmo modo que seus respectivos fatores, reportando a psicopatia primária como mais característica em relação à psicopatia secundária.

Conhecidos os escores médios dos construtos avaliados, utilizou-se o teste Kolmogorov-Smirnov para verificar quais apresentavam distribuição normal. Assim, observou-se que apenas as variáveis tempo de ressocialização $(z=0,22 ; p=0,20)$, agressividade física $(z=0,22 ; p=0,61)$, agressividade verbal $(z=0,16 ; p$ $=0,20)$ e psicopatia primária $(z=0,16 ; p=0,20)$ apresentaram distribuição normal.

Diante desses resultados, optou-se por utilizar a correlação $r$ de Pearson para aquelas variáveis que apresentaram distribuição normal. Desse modo, buscou-se compreender se os escores dessas dimensões apresentam relação com o tempo de inserção em atividade de ressocialização. Nesse caso, identificou-se que as dimensões de agressividade física $(\mathrm{r}=-0,20 ; p=0,20)$ e verbal $(\mathrm{r}=-0,05 ; p=0,76)$ não se apresentaram relacionadas com o tempo de inserção em atividade de ressocialização. Igualmente, constatou-se que o fator de psicopatia primária $(\mathrm{r}=0,11 ; p=0,47)$ não apresentou correlação com o tempo de inserção em atividade de ressocialização.

Ainda no intuito de verificar se o tempo de inserção em atividade de ressocialização guarda associação com os escores dos construtos avaliados, 
foram realizadas correlações rô de Spearman para as dimensões que não apresentaram distribuição normal. Os resultados indicaram não existir correlação entre os escores totais de agressividade $(\mathrm{r}=-0,10 ; p$ $=0,52)$, os fatores de raiva $(\mathrm{r}=-0,19 ; p=0,23)$ e hostilidade $(\mathrm{r}=0,07 ; p=0,64)$ com o tempo de inserção em atividade de ressocialização. Do mesmo modo, os escores totais de psicopatia $(r=0,06 ; p=0,96)$ e da dimensão psicopatia secundária $(\mathrm{r}=-0,03 ; p=0,81)$ não se apresentaram relacionados. Por fim, ainda que não seja o foco da presente pesquisa, optou-se por apresentar o padrão de correlação das variáveis aqui apresentadas (Tabela 3).

\section{Discussão}

O presente estudo teve como objetivo caracterizar fatores de risco, em específico a psicopatia, agressividade e a personalidade, em detentos inseridos em atividade de ressocialização. Os escores de agressividade e

Tabela 2

Média, Desvio Padrão, Minimo e Máximo, Erro Padrão e Intervalo de Confiança de 95\%

\begin{tabular}{lccccc}
\hline variáveis & média & desvio padrão & mínimo-máximo & Erro padrão & IC $95 \%$ \\
\hline agressivida total & 2,47 & 0,63 & $1,19-3,88$ & 0,09 & $2,28,2,65$ \\
agressividade física & 1,75 & 0,74 & $1-3,43$ & 0,10 & $1,53,1,97$ \\
agressividade verbal & 2,64 & 1,16 & $1-5$ & 0,16 & $2,30,2,98$ \\
raiva & 2,47 & 0,91 & $1-4,17$ & 0,13 & $2,20,2,74$ \\
hostilidade & 2,92 & 0,73 & $1,30-4,40$ & 0,10 & $2,70,3,13$ \\
\hline abertura & 4,16 & 0,60 & $2,56-5,00$ & 0,08 & $3,98,4,34$ \\
neuroticismo & 2,40 & 0,87 & $1-3,83$ & 0,12 & $2,15,2,66$ \\
extroversão & 3,37 & 0,55 & $2,13-4,5$ & 0,08 & $3,21,3,53$ \\
consienciosidade & 4,07 & 0,63 & $2,25-5$ & 0,09 & $3,88,4,25$ \\
amabilidade & 4,63 & 0,65 & $2,67-5$ & 0,09 & $4,44,4,83$ \\
\hline psicopatia primária & 2,02 & 0,57 & $1-3,41$ & 0,08 & $1,86,2,19$ \\
psicopatia secundária & 1,90 & 0,45 & $1-2,89$ & 0,06 & $1,77,2,03$ \\
psicopatia total & 1,89 & 0,48 & $1-3,15$ & 0,06 & $1,84,2,12$ \\
\hline
\end{tabular}

Tabela 3

Correlacões entre Psicopatia, Agressividade e Personalidade

\begin{tabular}{lcc}
\hline variáveis & psicopatia primária & psicopatia secundária \\
\hline agressividade física & $0,37^{* *}$ & $0,63^{* *}$ \\
agressividade verbal & $0,66^{* *}$ & $0,44^{* *}$ \\
raiva & $0,67^{* *}$ & $0,44^{* *}$ \\
hostilidade & $0,44^{* *}$ & $0,64^{* *}$ \\
\hline abertura & $-0,11$ & $0,36^{*}$ \\
neuroticismo & 0,21 & 0,11 \\
extroversão & $-0,28$ & $-0,06$ \\
consienciosidade & $-0,32$ & $-0,15^{*}$ \\
amabilidade & $-0,26$ & 0,26 \\
\hline
\end{tabular}

Nota. $* p<0,05 ; * * p<0,01$ 
psicopatia apresentaram-se convergentes com estudos prévios realizados em amostras de detentos, e o padrão de personalidade mostrou-se marcadamente adaptativo e divergente com estudos prévios realizados em amostras de universitários (Diamond \& Magaletta, 2006; Brinkley et al., 2001).

No entanto, chama a atenção o fato de que os escores de psicopatia e agressividade, aqui reportados, serem inferiores aos reportados em estudos com amostras de universitários (Coelho, Paixão, \& Silva, 2010, Brinkley et al., 2001). Ainda que inseridos em atividade de ressocialização, em contraste com amostras de universitários possivelmente expostos a fatores de risco, os resultados apontam ausência de relação dos escores reportados com o tempo de inserção em atividades de ressocialização. Tais resultados sugerem que a utilização de medidas de autorrelato pode ter influência na aferição de traços de psicopatia, indicando que indivíduos com traços psicopáticos mais acentuados podem manipular suas respostas para apresentarem escores inferiores aos esperados. Percebe-se, no entanto, que a dimensão de psicopatia primária, caracterizada também por uma postura manipuladora, apresenta maior escore nesse construto e que a dimensão de psicopatia secundária, marcada por uma postura impulsiva e de estilo de vida inapropriado, é reportada como um escore inferior.

Os resultados também apontam que, apesar da característica manipuladora, a amostra estudada tende a ser mais hostil e agressiva verbalmente. Considera-se que esse padrão de agressividade converge com a literatura, de modo que a agressividade verbal e a hostilidade apresenta-se mais proeminentes em indivíduos com traços psicopáticos (Patrick \& Zempolich, 1998; Stanford, Houston, Pittman, \& Grave, 2003). Contudo, os escores apresentados também são inferiores quando comparados a estudos que avaliaram universitários com o mesmo instrumento (Formiga et al., 2007; Formiga et al., 2010), corroborando com dominância da dimensão de psicopatia primária. Ainda nesse sentido, o padrão de agressividade corrobora com indicação de impulsividade da dimensão secundária da psicopatia.

No tocante à personalidade, o padrão apresentado sugere ser aparentemente adaptativo, indicando escores altos em amabilidade, abertura à mudança, conscienciosidade, extroversão e, como a única dimensão com escore inferior ao ponto médio da escala, o neuroticismo. Esse padrão é marcadamente divergente daqueles presentes na literatura, em estudantes e detentos, indicando uma manifestação de características socialmente desejáveis. Assim, quando esse padrão de personalidade é confrontado com estudos que buscaram relacionar traços de psicopatia e traços normais de personalidade em universitários, por meio dos mesmos instrumentos, aos achados, eles são divergentes. Miller, Gaughan e Pryor (2008) reportam, em uma amostra de universitários, uma relação negativa com o traço de amabilidade, conscienciosidade, extroversão, uma relação positiva com neuroticismo e ausência de relação com a dimensão de abertura à mudança. Um padrão semelhante também é apresentado quando utilizado o PCL-R (Lynam \& Derefinko's, 2006; Miller, Watts, \& Jones, 2011). Da mesma forma, quando comparado com a metanálise realizada por O`Boyle et al. (2014) os achados da amostra estudada são divergentes, indicando respostas possivelmente motivadas por desejabilidade social.

Diferentemente, Hart \& Hare (1994) investigaram a relação entre os escores de psicopatia de detentos, obtido por meio de autorrelato, e a classificação de observadores sobre traços normais da personalidade desses detentos e observaram um padrão mais próximo daquele aqui obtido, sendo reportadas relações positivas com extroversão, amabilidade, conscienciosidade, abertura à mudança e relação negativa com neuroticismo. Nesse contexto, aponta-se que, na amostra estudada, a configuração dos traços de personalidade coaduna com a indicação de um perfil pertinente com o socialmente desejável. Assim sendo, os resultados reportados apontam na direção oposta de estudos realizados previamente, mesmo aqueles que utilizaram de diferentes medidas de psicopatia e de traços normais da personalidade, apontando, na amostra estudada, que amabilidade prevalece como a dimensão com escores mais elevados.

Dessa forma, o aglomerado de resultados reportados se agrega a evidências prévias de descrições de amostras carcerárias no Brasil (Jozef et al., 2000; Schmitt et al., 2006). No que tange a marcante característica de desejabilidade social presente nos dados, pode-se apontar possíveis fatores que influenciaram as respostas: (1) a marcante tendência a mentir, manifesta em indivíduos com traços psicopáticos, pode indicar possíveis limitações nas avaliações realizadas por meio do autorrelato em casos práticos, como avaliação de probabilidade de violência futura e progressão de regime penal. Tais indícios também devem ser levados em consideração na interpretação de evidências baseadas em autorrelato para descrição da relação de traços de psicopatia com outros construtos. Mesmo que extrapolando os resultados aqui relatados, a exploração de uma perspectiva não criminosa do traço de psicopatia, indicando 
o comportamento criminoso como um correlato, pode apontar para uma capacidade adaptativa da estrutura de traços normais da personalidade quando avaliados por meio de autorrelato, ou seja, indivíduos com traços de psicopatia podem apresentar a capacidade de reportar padrões de traços normais da personalidade que melhor se adequem a seus objetivos e; (2) Apesar dessas características marcantes do traço de psicopatia, a utilização do autorrelato já é amplamente consolidada e reúne evidências sólidas nesse contexto. Assim, outro fator que pode ter influenciado a desejabilidade social é o funcionamento do sistema prisional com relação a pesquisa. Em geral, os pesquisadores devem seguir o modus operandi do sistema prisional, buscando interferir o mínimo possível na rotina do sistema, visando à segurança de todos os envolvidos, o que pode induzir o detento a compreender sua participação na pesquisa como uma forma de inquérito do sistema judiciário. $\mathrm{Na}$ presente pesquisa, o modus operandi do sistema prisional foi respeitado e os pesquisadores buscaram reduzir uma possível influência de um ambiente "coercitivo" por meio de algumas providências previamente acordadas com a direção do presídio, como: (a) a entrevista foi realizada em uma sala com condições climáticas e acústicas confortáveis; (b) o encaminhamento dos detentos para a sala de entrevista era realizado por um agente penitenciário que aguardava na antessala daquela onde a entrevista estava sendo realizada; (c) nenhum dos detentos utilizavam algemas ou qualquer acessório limitante de seus movimentos; (d) todas as entrevistas foram realizadas por meio de dois avaliadores que informaram sobre os objetivos da pesquisa e que os dados não seriam disponibilizados de modo individualizado para o sistema ou teriam qualquer influência no cumprimento da pena.

Apesar dos passos anteriormente descritos, não se descartam aqui possíveis influências advindas desse modus operandi, como o baixo nível de escolaridade encontrado, que pode ter influenciado na compreensão da utilização dos dados fornecidos, a presença de um segundo entrevistador que pode ter sido encarada como uma "testemunha" dos dados fornecidos ou características peculiares do tratamento dado aos detentos pelos agentes penitenciários que os encaminhavam para sala de entrevista.

Não obstante, deve-se ter parcimônia ao observar tais evidências. A presente pesquisa utilizou-se de uma amostra não probabilística de detentos inseridos em atividade de ressocialização, o que pode ter acarretado uma limitação da expressão do traço de psicopatia.
Ademais, a situação de cárcere, o nível econômico e a escolaridade podem estar presentem como variáveis de confusão. Além disso, como supracitado, a utilização de medidas de autorrelato e/ou o modus operandi do sistema prisional pode ter incorporado distorções ou enviesamentos das respostas decorrentes da situação de cárcere.

Por fim, recomenda-se que, em estudos futuros, a utilização de amostras provenientes de outros regimes no sistema prisional (semiaberto, fechado e liberdade condicional), utilizando-se de protocolos de pesquisa que, aliados ao modus operandi do sistema penitenciário, possa reduzir possibilidades de vieses como a desejabilidade social. Também se faz necessária a utilização de métodos de controle de desejabilidade social para os construtos aqui elencados, como o a versão do Big Five Inventory composta por itens com menor desejabilidade social (Costa \& Hauck-Filho, 2017), a utilização de medidas implícitas (Athayde, 2012) ou avaliações que possam reduzir o falseamento das respostas. Nesse sentido, psicopatas têm demonstrado características atípicas em respostas fisiológicas mediante tarefas, como antecipação de estímulos desagradáveis ou dolorosos, atenção, memória e estímulos estressantes (Huss, 2011; Blair, Jones, Clark, \& Smith, 1997). A investigação de tais estratégias no contexto brasileiro pode apresentar uma possibilidade no desenvolvimento de novas estratégias de avaliação mais eficientes e possibilitar a redução de respostas socialmente desejáveis. De tal modo, inserir esses procedimentos e métodos em pesquisas futuras parece ser de grande valor e importância para o entendimento do construto.

\section{Referências}

Andrade, J. M. (2008). Evidências de Validade do Inventário dos Cinco Grandes Fatores de Personalidade para o Brasil (tese não publicada). Brasilia, DF. Universidade de Brasilia. Recuperado de http:/ / repositorio.unb.br/ handle/10482/1751

Armani, T. E., \& Cruz-Silva, C. T. A. (2010). Avaliação socioeconômica e de fatores que levam a violência com detentos de Cascavel/PR. Ensaio: Avaliação e Políticas Públicas em Educação, 18(67), 253-274. doi: 10.1590/S0104-40362010000200005

Athayde, R. A. A. (2012). Medidas implicitas de valores bumanos: Elaboração e evidências de validade (dissertação de mestrado não publicada). Departamento de 
Psicologia da Universidade Federal da Paraíba, João Pessoa, PB.

Benet-Martinez, V. \& John, O. P. (1998). Los cinco grandes across cultures and ethnic groups: Multitrait multimethod analyses of the big Five in Spain and English. Journal os personality and social psychology, 75(3), 729-750. doi: 10.1037/0022-3514.75.3.729

Blair, E. B., Jones, L., Clark F., \& Smith, M. (1997). The psychopathy individual: A lack of responsiveness to distress cues? Psychophysiology, 34, pp.192-198. doi: 10.1111/j.1469-8986.1997.tb02131.x

Brasil, Ministério da Justiça. (2014). Sistema Penitenciário Nacional do Brasil. População carcerária sintética. Brasília: Departamento Penitenciário Nacional. Recuperado de http://s.conjur.com.br/dl/infopen-dez14.pdf

Brasil, Ministério da justiça. (2015). Levantamento nacional de informações penitenciárias. Brasília: Conselho Nacional de Justiça. Recuperado de http:// www.justica.gov.br/seus-direitos/politica-penal/ transparencia-institucional/estatisticas-prisional/ levantamento-nacional-de-informacoes-penitenciarias

Brinkley, C. A., Schmitt, W. A., Smith, S. S., \& Newman, J. P. (2001). Construct validation of a self-report psychopathy scale: does Levenson's self-report psychopathy scale measure the same constructs as Hare's psychopathy checklist-revised? Personality and individual differences, 31(21), pp. 1021-1038. doi: 10.1016/S0191-8869(00)00178-1

Brinkley, C. A., Diamond, P. M., Magaletta, P. R., \& Heigel, C. P. (2008). Cross-Validation of Levenson's Psychopathy Scale in a Sample of Federal Female Inmates. Assessment, 15(4), 464-482. doi: 10.1177/1073191108319043

Bushman, B. J., \& Anderson, C. A. (2001). Is it time to pull on the plug on the hostile versus instrumental, aggression dichotomy? Psychologicall Rewiew, 108, 273-279. doi: 0.1037/0033-295X.108.1.273

Buss, A. H., \& Perry, M. The aggression questionnaire. (1992). Journal of Personality and Social Psychology. 63, 452-459. doi: 10.1037/0022-3514.63.3.452

Coelho, L., Paixão, R., \& Silva, J. T. (2010). O Levenson's Self Report Psychopathy Scale (LSRP). Psychologica, 52, pp. 413-421. doi: 10.14195/1647-8606_53_20
Costa, A. R. L., \& Hauck Filho, N. (2017). Menos desejabilidade social é mais desejável: Neutralização de instrumentos avaliativos de personalidade. Temas em Psicologia, 21(3), 239-249. doi: 10.5380/psi. v21i3.53054

Costa, P. T., \& Widiger, T. A. (1993). Introduction. Em P. T. Costa \& T. A. Widiger (Ed.), Personality Disorders and the five-factor model of personality (pp. 1-10). Washington, DC: American Psychological Association.

Diamond, P. M., \& Magaletta, P.R. (2006). The Short-Form Buss-Perry Aggression Questionnaire (BPAQ-SF). A Validation Study With Federal Offenders. Assessment, 13 (3). doi: $0.1177 / 1073191106287666$

Formiga, S. N., Cavalcante, C. P., Araújo T. T. V., Lima, S., \& Santana, R. (2007). Comportamento Agressivo e Busca de Sensação em Jovens. Psicologia e Argumentação, 25(50). Recuperado de http://132.248.9.34/hevila/Psicologiaargumento $/ 2007 /$ vol25/no50/6.pdf

Formiga, N. S., Omar, A. C., \& Aguiar, M. (2010). Busca de sensação e uso potencial de drogas em universitários brasileiros. Psicologia Revista. 19 (1), 97-118. Recuperado de https://revistas.pucsp.br/index. $\mathrm{php} / \mathrm{psicorevista/article/view/5222}$

Gouveia, V. V., Chaves, C. M. C. M., Peregrino, R. R., Branco, A. O. C., \& Gonçalves,

M. P. (2006). Medindo componentes da agressão: o questionário de Buss-Perry. Arquivos brasileiros de psicologia, 60(3), 92-103. Recuperado de http:// seer.psicologia.ufrj.br/index.php/abp/article/ view/207/253

Hare, R.D. (1991). The Hare Psychopatby Checklist Revised. Toronto, Canadá: Multi-Health Systems.

Hare, R. D. (2003). The Hare Psychopathy Checklist. Second Edition. Toronto, Canadá: Multi-Health Systems.

Hare, R. D. (2013). Sem consciência: O mundo perturbador dos psicopatas que vivem entre nós. Porto Alegre: Artmed.

Hart, S. D., \& Hare, R. D. (1994). Psychopathy and The Big 5: Correlations Between Observers' Ratings of Normal and Pathological Personality. Journal of Personality Disorders, 8(1), 32-40. doi: 10.1521 
Hauck-Filho, N., \& Teixeira, M. A. P. (2014). Revisiting the psychometric properties of the Levenson self-report psychopathy scale. Journal of Personality Assessment, 96(4), 459-64. doi: 10.1080/00223891.2013.865196

Hauck Filho, N., Teixeira, M. A. P., \& Dias, A. C. G. (2012). Psicopatia: Uma perspectiva dimensional e não criminosa do construto. Avances en Psicología Latinoamericana, 30(2) 317-327. Recuperado de http://web.a.ebscohost.com/abstract?direc$\mathrm{t}=$ true\&profile $=$ ehost\&scope $=$ site $\&$ authtype $=$ crawler\&jrnl $=17944724 \& A N=89060151 \& \mathrm{~h}=\mathrm{L}$ uJSdAUID475ongrZREI9pL1 tBCc\%2fXLG5i B17aIwcZAGc791X8xw2uzUszeILyxpAAorE1t M07azx3Y9A7cXTQ\%3d\%3d\&crl=c\&resultN $\mathrm{s}=$ AdminWebAuth\&resultLocal=ErrCrlNotAu th\&crlhashurl=login.aspx $\% 3$ fdirect $\% 3$ dtrue $\% 2$ 6profile $\% 3$ dehost $\% 26$ scope $\% 3$ dsite $\% 26$ authty pe\%3dcrawler\%26jrnl\%3d17944724\%26AN\% 3d 89060151

Hershcovis, M. S., Turnr, N., Barling, J., Arnold, K. A., Dupré, K. E., Inness, M., Leblanc, M. M., \& Sivanathan, N. (2007). Predicting Workplace Aggression: A Meta-Analysis. Journal of Applied Psychology. 92(1), 228-238. doi: 10.1037/0021-9010.92.1.228

Huss, M. T. (2011). Psicologia Forense: Pesquisa, prática clínica e aplicaşões. Porto Alegre: Artmed.

Jhon, O. P., Donahue, E. M., \& Kentle, R. L. (1991). The Big Five Inventory - Version $4^{a}$ and 54. Berkeley, CA: University of California, Berkeley, Institute of Personality and Social Research.

Jozef, F., Silva, A. R., Greenhalgh, S., Leite, M. E., \& Ferreira, V. H. (2000). Comportamento violento e disfunção cerebral: Estudo de homicidas no Rio de Janeiro. Revista Brasileira de Psiquiatria, 22(3), 124129. doi: 10.1590/S1516-44462000000300005

Lago, V. M., \& Bandeira, D. R. (2008). As práticas em avaliação psicológica envolvendo disputa de guarda no Brasil. Avaliação Psicológica, 7(2), 223-234. Recuperado de http://pepsic. bvsalud.org/scielo.php?script=sci_arttext\&pi$\mathrm{d}=$ S1677-04712008000200013\&lng=pt\&tlng=pt.

Levenson, M. R., Kiehl, K. A., \& Fitzpatrick, C. M. (1995). Assessing psychopathic attributes in a noninstitutionalized population. Journal of Personality and Social Psychology, 68(1), 151-158. doi: 10.1037/0022-3514.68.1.151
Levenson, J. S. (2004). Sexual Predator Civil Commitment: A Comparison of Selected and Released Offenders. International Journal of Offender Therapy and Comparative Criminology, 48(6), 2004 638-648. doi: 10.1177/0306624X04265089

Listwan, S. J., Piquero, N. L., \& Voorhis, P. V. (2010). Recidivism Among a White-Collar Sample: Does Personality Matter? The Australian And New Zealand Journal Of Criminology, 43(1), 156-174. doi: 10.1375/acri.43.1.156

Lynam, D. R., \& Derefinko, K. J. (2006). Psychopathy and Personality. Em C. J. Patrick (Ed.), Handbook of Psychopathy (pp. 133-155). New York: The Guilford Press.

McCrae, R. R., \& John, O. P. (1992). An introduction to the five-factor model and its applications. Journal of Personality, 60, 175-215. doi: 10.1111/j.14676494.1992.tb00970.x

McCrae, R. R. (2006). O que é personalidade? Em C. Flores-Mendonza \& R. Colom (Ed.), Introdução à psicologia das diferenças individuais (pp. 203-218). Porto Alegre: Artmed.

Miller, J. D., Gaughan, E. T., \& Pryor, L. R. (2008). The Levenson Self-Report Psychopathy scale: An examination of the personality traits and disorders associated with the LSRP factors. Assessment, 15(4), 450-63. doi: 10.1177/1073191108316888

Miller, J. D., Lynam, D., \& Leukefeld, C. (2003). Examining antisocial behavior through the lens of the five factor model of personality. Aggressive Behavior, 29, 497-514. doi: 10.1002/ab.10064

Miller, J. D., Watts, A., \& Jones, S. E. (2011). Does psychopathy manifest divergence relations with componentes of its nomological network depending on gender? Personality and Individual Differences, 50, 564-69. doi: 10.1016/j.paid.2010.11.028

Monteiro, R. P., Gouveia, R. S. V., Patrick, C. J., Medeiros, E. D., Pimentel, C. E., \& Gouveia, V. V. (2015). A psicopatia no contexto dos cinco grandes fatores. Psico. 46(4), 461-471. doi: 10.15448/1980-8623.2015.3.20314

O'Boyle, E. H., Forsyth, D. R., Banks, G. C., Story, P. A., \& White, C. D. (2014). A Meta-Analytic Test of Redundancy and Relative Importance of the Dark Triad and Five-Factor Model of Personality. Journal of Personality, n/a-n/a. doi: 10.1111/jopy.12126 
Patrick, C. J., \& Zempolich, K.A. (1998). Emotion and aggression in the psychopathic personality. $A g$ gression and Violent Behavior. 3(4), pp. 303-338. doi: 10.1016/S1359-1789(97)00003-7

Penney, S. R., \& Moretti, M. M. (2007). The relation of psychopathy to concurrent aggression and antisocial behavior in high-risk adolescent girls and boys. Behavioral Sciences and the Law, 25, 21-41. doi: 10.1002/bsl.715

Pervin, L. A., \& John, O. P. (2004). Personalidade: Teoria e pesquisa, $8^{a} e d$. Porto Alegre: Artmed Editora.

Schippers, G. M., Märker, N., \& Fuentes-Merillas, L. (2001). Social Skills Training, Prosocial Behavior, and Aggressiveness in Adult Incarcerated Offenders. International Journal of Offender Therapy and Comparative Criminology, 45(2), 244-251. doi: 10.1177/0306624X01452009

Schmitt, R., Pinto, T. P., Gomes, K. M, Quevedo, J., \& Stein, A. (2006). Personalidade psicopática em uma amostra de adolescentes infratores brasileiros. Revista de Psiquiatria Clínica, 33(6), 297-303. doi: 10.1590/S0101-60832006000600002

Schraft, C. V., Kosson, D. S., \& Mcbride, C. K. (2013). Exposure to violence within home and community environments and psychopathic tendencies in detained adolescents. Criminal justice and behavior. 40(9), 1027-1043. doi: 10.1177/0093854813486887

Silva, M. C. R., \& Fontana, E. (2011). Psicologia Jurídica: Caracterização da prática e instrumentos utilizados. Estudos Interdisciplinares em Psicologia, 2(1), 56-71. Recuperado de http://pepsic. bvsalud.org/scielo.php?script $=$ sci_arttext\&pi$\mathrm{d}=$ S2236-64072011000100005\&lng=pt\&tlng=pt.

Smith, P. N., Selwyn, C. N., Wolford-Clevenger, C., \& Mandracchia, J. T. (2014) Psychopathic personality traits, suicide ideation, and suicide attempts in male prison inmates. Criminal Justice And Behavior, 41(3), 364-379. doi: 10.1177/0093854813506884
Sobral, J., Romero, E., Luengo, A., \& Marzoa, J. (2000). Personalidad y conducta antisocial: Amplificadores individuales de los efectos contextuales. Psicothema, 12, 661-670. Recuperado de http://www.psicothema.com/pdf/387.pdf

Stanford, M. S., Houston, R. J., Villemarette-Pittman, N. R., \& Greve, K. W. (2003). Premeditated aggression: Clinical assessment and cognitive psychophysiology. Personality and Individual Differences, 34, 773-781. doi: 10.1016/S0191-8869(02)00070-3

Swogger, M. T., Walsh, Z., Lejuez, C. W., \& Kosson, D. S. (2010). Psychopathy and risk taking among jailed inmates. Criminal Justice And Behavior, 37(4), 439-452. doi: 10.1177/0093854810361617

Tóth, M., Halász, J. Mikics É., Barsy, B., \& Halle, J. (2008). Early social deprivation induces disturbed social communication and violent aggression in adulthood. Behavioral Neuroscience, 122(4), 849-854. doi: 10.1037/0735-7044.122.4.849.

Vachon, D. D., Lynam, D. R., Widiger, T. A., Miller, J. D., McCra, R. R., \& Costa, P. T. (2013). Basic traits predict the prevalence of personality disorder across the life span: the example of psychopathy. Psychol Science, 24(5), 698-705. doi: $10.1177 / 0956797612460249$

Vargas, F., Hoffmeister, F. X., Prates, P. F., \& Vasconcelos, S. J. L. (2015). Depressão, ansiedade epsicopatia: Um estudo correlacional com indivíduos privados de liberdade. Jornal Brasileiro de Psiquiatria, 64(4), 266-71. doi: 10.1590/0047-2085000000088

Walnsley,R.(2013).WorldPrisonPopulationList.International CenterforPrison Studies, 10ed.Victoria Charity. Recuperado de http://www.prisonstudies.org/resources/ world-prison-population-list-10th-edition

Recebido em: 16-10-2017

Reformulado em: 03-04-2018

Aprovado em: 03-05-2018 
Nota dos autores:

Os autores agradecem a Fundação de Amparo a Pesquisa do Estado de Alagoas - FAPEAL, pelo apoio financeiro na modalidade bolsa mestrado concedida ao primeiro autor.

Sobre os autores:

Germano Gabriel Lima Esteves é psicólogo (UFAL), mestre em Psicologia (UFAL), doutorando em Psicologia Social, do Trabalho e das Organizações (PSTO - UnB), professor adjunto I na Universidade de Rio Verde (UniRV) e coordenador do Laboratório de Avaliação Psicológica de Rio Verde (LAPRV).

E-mail: germanoesteves@unirv.edu.br

Id: https:/ / orcid.org/0000-0002-1851-4603

Jorge Artur Peçanha de Miranda Coelho é doutor em Psicologia Social (2009) pela Universidade Federal da Paraíba e professor adjunto da Universidade Federal de Alagoas (UFAL).

E-mail: jorge.coelho@famed.ufal.br

Id: https:/ / orcid.org/0000-0002-0021-5963

Bruna Nogueira Romariz Barros é psicóloga e mestranda em Ciências da Saúde pela Universidade Federal de Alagoas.

E-mail:brunanromariz@gmail.com

Id: https:/ / orcid.org/0000-0001-9999-7522

Gustavo Henrique Silva de Souza é mestre em Psicologia e bacharel em Administração pela Universidade Federal de Alagoas (UFAL), professor e pesquisador do Instituto Federal do Norte de Minas Gerais (IFNMG), editor associado do Journal of Education, Sciety and behavioural science e líder do grupo de Pesquisa Práticas da Administração Eupsíquica, Bases do Comportamento Organizacional e Medidas Psicométricas.

E-mail: souza.g.h.s@gmail.com

Id: orcid.org/0000-0002-4046-9669

Contato com os autores:

Universidade de Rio Verde, Campus Rio verde, Laboratório de Avaliação Psicológica de Rio Verde - LAPRV

Rio Verde-GO, Brasil

CEP: 75901-970 